\title{
Effect of starch supply to the rumen on volatile fatty acids, lactate and $D \beta$-hydroxybutyrate net fluxes in the ruminal veins of sheep fed hay
}

\author{
D Rémond, E Delval, JP Chaise
}

\author{
INRA, SRNH, Theix, 63122 St-Genès-Champanelle, France
}

Three wethers were surgically fitted with catheters in the ruminal veins and in a mesenteric artery, blood flow probes around ruminal arteries, and a ruminal cannula. They were fed $500 \mathrm{~g}$ of orchardgrass hay every $12 \mathrm{~h}$, and received successively, for at least $10 \mathrm{~d}$ before measurements, three levels of wheat starch $(0,120,240 \mathrm{~g} / 12 \mathrm{~h})$ directly injected into the rumen during the meal. Eight sets of venous and arterial blood and rumen content were collected within the feeding cycle. Blood flow was measured with an ultrasonic transit time flowmeter. Sample analysis and metabolite net fluxes calculation were conducted as previously described by Rémond et al (1993, J Anim Sci, 71, 2529-2538). The effects of starch supply were statistically analysed using repeated measures analysis of variance.

Starch fermentation increased propionate and butyrate concentration in the rumen and decreased acetate: propionate ratio. Propionate net release into the ruminal veins was linearly related to the increase in propionate concentration in the rumen $(\mathrm{P}<0.01$, $\mathrm{r}^{2}=0.83, \mathrm{n}=9$ ). Part of the lactate production in the ruminal epithelium is supposed to originate from propionate metabolism, however no change in lactate net release was detected in the present study despite the increase in propionate net release. Increasing the supply of propionate in the rumen, Seal and Parker (1994, J Anim Sci, 72, 1325-1334) observed that the increase in propionate net release was associated with an increase in lactate net release from the forestomach of sheep. The discrepancy between their observations and ours could be explain by a limitation of propionate metabolism due to the inhibitory effect of butyrate on propionyl-CoA in the ruminal epithelium, and by an increase in the oxydation rate of the metabolised propionate as observed with grain diet (Harmon et al, 1991, J Anim Sci, 69, 4117-4127).

The main substrate for hydroxybutyrate (D $\beta \mathrm{HOB}$ ) production in the ruminal epithelium is butyrate. Although butyrate net release increased with starch supply, DBHOB net release decreased. Krehbiel et al (1992, J Anim Sci, 20, 904-914) previously reported that an increase in ruminal butyrate produced an increase in butyrate net release in portal blood not associated with an increase in $\mathrm{D} \beta \mathrm{HOB}$ production. Furthermore Seal and Parker (1994, J Anim Sci, 72, 1325-1334) observed a decrease in $\mathrm{D} \beta \mathrm{HOB}$ net release in portal blood when propionate absorption was increased. Therefore the decrease in $D \beta H O B$ production in the ruminal epithelium observed could result both, from the limited capacity of the epithelium to produce ketone body, and from an antiketogenic effect of propionate.

These results underline the influence of the interactions between VFA on the metabolism of the ruminal epithelium in vivo.

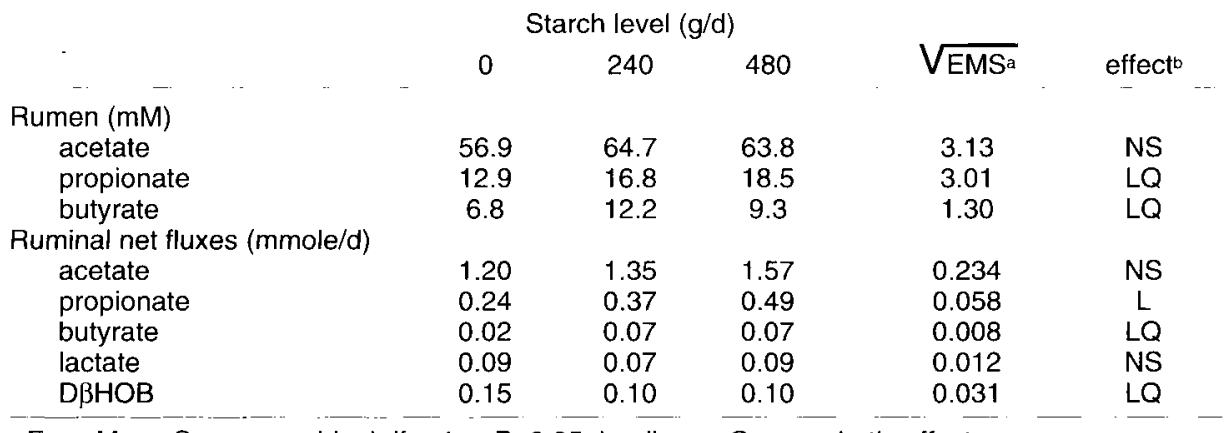

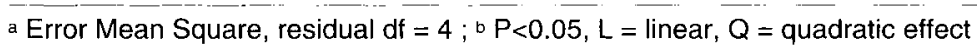

